\title{
L'impossible " chambre des horreurs " du Museum of Ornamental Art : une archéologie du design criminel
}

\section{Marie-Ève Marchand}

Volume 39, numéro 1, 2014

URI : https://id.erudit.org/iderudit/1026201ar

DOI : https://doi.org/10.7202/1026201ar

Aller au sommaire du numéro

\section{Éditeur(s)}

UAAC-AAUC (University Art Association of Canada | Association d'art des universités du Canada)

ISSN

0315-9906 (imprimé)

1918-4778 (numérique)

Découvrir la revue

Citer cet article

Marchand, M.-È. (2014). L'impossible " chambre des horreurs » du Museum of Ornamental Art : une archéologie du design criminel. RACAR : Revue d'art canadienne / Canadian Art Review, 39(1), 40-51.

https://doi.org/10.7202/1026201ar

\section{Résumé de l'article}

In 1852, the Museum of Ornamental Art, today the Victoria and Albert Museum, opened its doors to the public. Taking part in a general reform of the British art and design education system, the museum sought to instill what were considered good design principles. To do so, a museographic strategy that proved to be as popular as it was controversial was chosen: the exhibition gallery entitled "Decorations on False Principles," which immediately became known as the "Chamber of Horrors." This gallery, a dogmatic expression of the functionalist conception of ornament advocated by the museum, referred through its nickname to another then famous Chamber of Horrors, the one in Mme Tussaud's wax museum. In this paper, I will first argue that the Museum of Ornamental Art's Chamber of Horrors is an early example of the association of ornament with crime that reappears in later design theories. Second, by examining the means taken to transmit the idea of the criminalization of ornaments designed after "bad principles," I demonstrate why the concept of the Chamber of Horrors is in itself doomed to failure. I thus analyze this uncommon exhibition as a manifestation of the museum's aesthetic philosophy and mechanisms at a time when the institution's modalities were still in the process of elaboration.
Tous droits réservés (C) UAAC-AAUC (University Art Association of Canada | Association d'art des universités du Canada), 2014
Ce document est protégé par la loi sur le droit d'auteur. L’utilisation des services d'Érudit (y compris la reproduction) est assujettie à sa politique d'utilisation que vous pouvez consulter en ligne.

https://apropos.erudit.org/fr/usagers/politique-dutilisation/ 


\title{
L'impossible « chambre des horreurs » du Museum of Ornamental Art : une archéologie du design criminel
}

\author{
Marie-Ève Marchand, Université de Montréal
}

\begin{abstract}
In 1852, the Museum of Ornamental Art, today the Victoria and Albert Museum, opened its doors to the public. Taking part in a general reform of the British art and design education system, the museum sought to instill what were considered good design principles. To do so, a museographic strategy that proved to be as popular as it was controversial was chosen: the exhibition gallery entitled "Decorations on False Principles," which immediately became known as the "Chamber of Horrors." This gallery, a dogmatic expression of the functionalist conception of ornament advocated by the museum, referred through its nickname to another then famous Chamber of Horrors, the one in Mme Tussaud's wax museum. In this paper, I will first argue that the Museum of Ornamental Art's Chamber of Horrors is an early example of the association of ornament with crime that reappears in later design theories. Second, by examining the means taken to transmit the idea of the criminalization of ornaments designed after "bad principles," I demonstrate why the concept of the Chamber of Horrors is in itself doomed to failure. I thus analyze this uncommon exhibition as a manifestation of the museum's aesthetic philosophy and mechanisms at a time when the institution's modalities were still in the process of elaboration.
\end{abstract}

L ondres, lundi 6 septembre 1852. Le Museum of Ornamental Art, aujourd'hui Victoria and Albert Museum, ouvre ses collections au public ${ }^{1}$. Officiellement intitulée "Decorations on False Principles ", la toute première salle d'exposition à travers laquelle doit circuler le visiteur sera rapidement mieux connue sous le nom de "Chamber of Horrors ». Ce surnom évocateur lui est attribué le jour même de l'ouverture de l'exposition dans un article paru dans le quotidien The Times et dont l'auteur anonyme pourrait fort bien être Henry Cole (1808-1882), l'un des principaux instigateurs et le premier directeur de l'institution ${ }^{2}$. À la toute fin du compte rendu de sa visite au musée, l'auteur explique :

The ante-room, is fitted up as a sort of « chamber of horrors ", with a collection of all kinds of so-called ornamental manufacture, which are considered to exhibit false principles of decoration [...]. ${ }^{3}$

Dans cette antichambre, quatre-vingt-sept objets conçus d'après des principes de design jugés mauvais (wrong) ou faux (false) sont exposés ${ }^{4}$. Mise en scène unique dans un musée des arts décoratifs, et aussi populaire que controversée, la « chambre des horreurs » est démantelée dès juin 1853, probablement en raison des protestations de la part des manufacturiers dont les objets y étaient présentés 5 .

Quelles sont les implications du surnom provocateur " chambre des horreurs " pour les objets qui y sont exposés? Quels sont les enjeux, pour l'institution elle-même et pour ses collections, d'une telle mise en exposition ? En répondant à ces deux questions, nous soutiendrons non seulement que, à travers la critique du mauvais design qui y est formulée, la " chambre des horreurs " suggère pour la première fois l'idée que l'ornement peut être " criminel ", mais aussi que les convictions esthétiques à l'origine de cette idée et les moyens mis en ouvre pour la concrétiser au musée sont à l'origine même de l'échec de cette mise en exposition'6.
Nous commencerons par rappeler les ambitions pédagogiques du Museum of Ornamental Art, posant ainsi la principale condition de possibilité de la " chambre des horreurs ", soit la croyance en des principes universels de bon design. En effet, c'est sur l'existence de ces principes que s'appuie la mission pédagogique du musée, mission dont la " chambre des horreurs" est l'une des stratégies. Puis, nous démontrerons que, parce qu'il fait référence à la " chambre des horreurs » du musée de Mme Tussaud, le surnom attribué à cette salle d'exposition associe pour la première fois les principes de design et la notion de crime dans un contexte muséal, une idée marquante dans le domaine du design et dont les effets au Museum of Ornamental Art n’ont jamais été analysés jusqu’à maintenant. Ensuite, nous établirons que, comme tentative d'institutionnalisation de la criminalisation de l'ornement, le principe même de la « chambre des horreurs » dans le musée d'arts décoratifs est voué à l'échec, et ce, indépendamment des raisons officielles ayant mené à sa fermeture. Pour ce faire, nous relèverons les failles du principal moyen muséographique déployé pour transmettre les principes de bon design dans cette salle : l'illustration par le contre-exemple. Si le recours au contre-exemple permet à l'institution de prodiguer son enseignement sur le mode visuel, la présentation visuelle d'un argument ne suffit toutefois pas à le rendre intelligible et se révèle plutôt source de confusion. Finalement, nous démontrerons que les ambiguïtés générées par la présentation de la « chambre des horreurs " au musée entraînent un risque de contamination qui remet en question l'autorité institutionnelle et marque la faillite de cette stratégie de mise en exposition.

L'histoire du Victoria and Albert Museum a fait l'objet de nombreuses études et la " chambre des horreurs " est mentionnée dans plusieurs de celles-ci ${ }^{7}$. Toutefois, à ce jour, seuls deux auteurs en proposent un examen détaillé. Avec son ouvrage Henry Cole and The Chamber of Horrors (2010), Christopher Frayling retrace à la fois le contexte d'origine, le contenu et l'histoire de cette salle d'exposition. Pour sa part, dans son 
article « Designing the Morality of Consumption : "Chamber of Horrors" at the Museum of Ornamental Art, 1852-53 ", Suga Yasuko démontre comment, parce qu'il unit étroitement le bon goût et la morale, le projet de la " chambre des horreurs " peut être associé au discours sur la consommation professé par les réformateurs du design en Angleterre au dix-neuvième siècle8.

Sans grande surprise, les principes de bon design ont pour corollaire le bon goût dans la société britannique de l'époque victorienne. Toutefois, notre ambition n'est pas ici d'inscrire la " chambre des horreurs " dans une histoire du goût en Angleterre au dix-neuvième siècle. Une telle démarche nécessiterait par exemple, comme l'a fait Francis Haskell avec Patrons and Painters (1963), d'examiner les rapports complexes entre les artistes et leurs commanditaires de manière à retracer l'ordre social, économique et politique dominant au sein duquel le goût pour l'art et la formation du canon sont - la plupart du temps - l'expression de la richesse et du pouvoir d'une classe au sommet de la hiérarchie sociale. Aussi, nous avons plutôt choisi d'analyser le mode de transmission des principes de bon design privilégié par le musée comme la formulation dogmatique d'une position fonctionnaliste à propos de l'ornement. Notre étude examine pour la première fois la " chambre des horreurs " en tant que manifestation de la philosophie esthétique du $\mathrm{Mu}$ seum of Ornamental Art de Londres et de ses mécanismes à une époque où les modalités de l'institution sont encore en processus d'élaboration.

\section{L'enseignement au Museum of Ornamental Art: une question de principe}

C'est sous la direction de Henry Cole qu'ouvre en 1852 le Museum of Ornamental Art. Le musée occupe alors le premier étage de la Marlborough House située à proximité du Palais de Buckingham et construite pour la duchesse de Marlborough au début du dix-huitième siècle d'après des plans de l'architecte Christopher Wren, lieu qu'il partage avec la School of Design'9. Déménagé sur le site de South Kensington en 1857, il devient le South Kensington Museum avant que l'appellation Victoria and Albert Museum ne lui soit attribuée en 1899. Grâce à une somme de 5000 livres allouée par le gouvernement britannique, le premier noyau des collections est acquis lors de la Great Exhibition of the Works of Industry of all Nations tenue à Londres en 1851. Sous la responsabilité d'un comité d'acquisition formé de Henry Cole, Richard Redgrave, Owen Jones et Augustus Welby Northmore Pugin, 244 objets dont bijoux, céramiques, émaux, mobilier, orfèvrerie, productions en papier mâché, tapis, textiles variés et verrerie sont acquis. D'autres pièces achetées quelques années plus tôt pour la School of Design et des prêts de collectionneurs complètent l'ensemble des objets présentés aux visiteurs ${ }^{10}$.
La mission du Museum of Ornamental Art est fondamentalement éducative ${ }^{11}$. L'ouverture de l'institution s'inscrit dans un projet de réforme générale du système de l'enseignement des arts piloté par le Department of Practical Art, une instance gouvernementale administrée par Cole et dont relève la gestion du musée ${ }^{12}$. Cette réforme vise entre autres à inculquer les principes de base pour juger correctement la valeur esthétique d'un objet ${ }^{13}$. Il s'agit ici de transmettre ce que l'institution considère comme les principes de bon design dont plusieurs sont, comme l'indique Clive Wainwright, tirés de l'ouvrage de Pugin, The True Principles of Pointed or Christian Architecture (1841) ${ }^{14}$.

L'un des buts de cette démarche est notamment d'améliorer la qualité des productions manufacturières du pays. Les décennies précédant l'ouverture du musée sont marquées, d'une part, par l'importation accrue, et à des prix plus abordables, d'objets de luxe d'origine française et, de l'autre, par un manque d'intérêt pour les produits britanniques sur le marché de l'exportation ${ }^{15}$. D'après un raisonnement favorisé par les opportunités offertes par la Révolution industrielle et afin d'encourager la production et la consommation d'objets qui, selon leurs critères, adopteraient des principes de bon design, les dirigeants du musée croient qu'il est essentiel d'éduquer à la fois les étudiants des écoles de design, les producteurs manufacturiers et le public.

Le précepte esthétique général qui se dégage des principes de bon design adoptés par l'institution et résumés dans le $\mathrm{Ca}$ talogue of the Museum of Ornamental Art de 1853 est celui de la subordination de l'ornement. Ce dernier doit être adapté à la fonction de l'objet ainsi qu'à son matériau. Par exemple, les motifs décoratifs doivent être petits sur les tissus épais comme les vichys, plus grands sur les mousselines ou encore espacés les uns des autres sur les cotons ${ }^{16}$. Par ailleurs, l'imitation directe de la nature est proscrite et il convient plutôt de s'inspirer de son fonctionnement. Ainsi, parce qu'un tapis est destiné à être déposé au sol et à servir de support à d'autres objets, ses ornements doivent être répartis uniformément sur sa surface et il faut éviter tout effet de tridimensionnalité (ombres portées, emploi de la perspective) ainsi que tout détail illusionniste tiré de la nature. En effet, qui, comme le raillaient certains observateurs de l'époque, oserait marcher sur un parterre fleuri ${ }^{17}$ ?

Sans qu'elle soit formulée en ces termes, nous voyons dans cette conception de l'ornement une forme de protofonctionnalisme, c'est-à-dire une position ayant une connotation fonctionnaliste avant la lettre. Bien que cette doctrine, dont l'enjeu ultime sera l'adéquation parfaite entre la forme et la fonction, soit surtout associée à la pratique de l'architecture et du design au vingtième siècle, certains de ses fondements sont posés dès le dix-neuvième. Dans The True Principles of Pointed or Christian Architecture, Pugin esquisse déjà une théorie de la relation entre la forme et la fonction. Pour lui, la première règle à suivre pour 


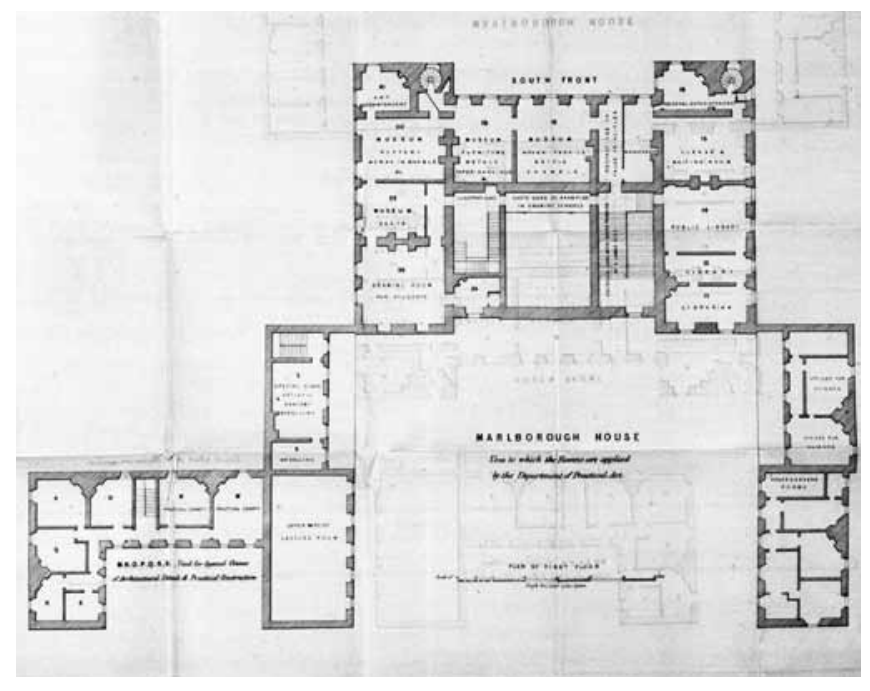

Figure I. Museum of Ornamental Art, plan du premier étage, I853, lithographié par Day and Son. Tiré de « Appendix VI (I.) : Marlborough House, Uses to which the Rooms are applied by the Department of Practical Art », First Report of the Department of Practical Art, Londres, 1853 (Photo : @ Victoria and Albert Museum, London).

bien concevoir un bâtiment est : " there should be no features about a building which are not necessary for convenience, construction, or propriety ${ }^{18}$. La tendance au fonctionnalisme s'observe également dans l'épuration et la rationalisation formelle opérées par certains concepteurs de mobilier comme Michael Thonet avec sa chaise bistrot (1859). Mais surtout, la conception fonctionnaliste de l'ornement au Museum of Ornamental Art fait la promotion de la spécificité de l'art ornemental. Régi par des principes de design qui lui sont propres et dont certains - refus de la perspective et du détail illusionniste notamment - s'opposent aux conventions traditionnelles de l'art pictural, l'art ornemental est ainsi posé comme étant distinct des "beaux arts".

L'un des éléments essentiels à la poursuite de l'objectif éducatif prôné par les responsables du musée est le fait qu'ils considèrent les principes de bon design comme étant transcendants. En effet, comme ces principes se retrouvent, selon eux, dans les meilleures œuvres de toutes les périodes ${ }^{19}$, ils seraient objectifs, voire universels. C'est ainsi que les visiteurs du musée sont encouragés à examiner les beautés et les défauts des objets exposés plutôt que de les considérer comme de simples curiosités ou objets de «vert $\hat{u} » 20$.

Les objets exposés au musée ont donc pour fonction d'exemplifier les principes de bon design prônés par l'institution. De l'aveu de Jones, il est difficile de traduire en mots les subtilités de ces principes, et ce, même pour les artistes qui les ressentent sans toutefois pouvoir les expliquer ${ }^{21}$. Les principes de bon design sont ainsi instanciés et matérialisés par des objets qui en deviennent l'illustration. L'emploi fréquent des mots specimen, example et illustration dans les descriptions publiées dans les catalogues de 1852 et 1853 confirme le rôle illustratif conféré à l'objet ${ }^{22}$. Grâce à la soi-disant universalité des principes de bon design qu'il prône et érige en dogme, le musée peut ainsi justifier la légitimité de sa mission, mais aussi ses méthodes pédagogiques. De fait, comme nous le verrons, le caractère absolu accordé aux principes de bon design encourage la transmission de l'argument esthétique sur le mode visuel au moyen de l'objet exemple. Véritable condition de possibilité de la criminalisation de l'ornement, ce caractère absolu autorise la présence de la " chambre des horreurs " qui, en retour, en devient l'expression paroxystique.

\section{La « chambre des horreurs », ou le design criminel}

Les objets exposés au Museum of Ornamental Art sont répartis entre cinq salles principales complétées d'une galerie de circulation où sont présentés des moulages en plâtre, et de l'escalier où sont accrochés des textiles de grandes dimensions et des carreaux de céramique. Étroite comme un corridor en raison de l'ajout d'une cloison, la première salle par laquelle le visiteur doit obligatoirement passer mesure environ 7 pieds de largeur par 23 pieds de profondeur. Dans le First Report of the Department of Practical Art, Henry Cole explique que les objets rassemblés dans cette salle sont conçus selon des principes de design qui se détournent de ceux jugés valables par les responsables du musée, d'où son intitulé officiel «Decorations on False Principles» (fig. 1) $)^{23}$.

Peu d'éléments nous sont parvenus à propos des quatrevingt-sept objets présentés dans cette salle et parmi lesquels se retrouvent principalement des tapis, des cotons imprimés, du papier peint, de la verrerie, des ouvrages de métal et de la céramique (fig. 2). Bien que seuls dix-sept d'entre eux aient été retracés, plusieurs de ces objets, notamment les textiles et les papiers peints, peuvent très certainement être comptés au nombre des produits destinés à la consommation de masse et qui, comme le remarque Lara Kriegel, inondent le marché des biens domestiques après avoir surchargé les cours du Crystal Palace en $1851^{24}$. Le contenu de la " chambre des horreurs " n'était donc vraisemblablement pas constitué d'objets uniques ou de prêts de collectionneurs, mais plutôt d'achats faits par le musée et dont la grande popularité de certains, le pichet numéro 76 , le plateau de papier mâché numéro 79 , le support pour rideaux numéro 80 ou la lampe au gaz numéro 83 (fig. 3) par exemple, est d'ailleurs soulignée dans le catalogue du musée publié en 185325 . Leur mode précis de présentation demeure toutefois inconnu et il n'existe aucune représentation visuelle de cette salle d'exposition. 
Bien que l'expression "chambre des horreurs " ne se retrouve pas dans le First Report of the Department of Practical Art, premier rapport annuel de l'institution rédigé principalement par Henry Cole, aucun indice ne laisse croire que cette épithète familière aurait pu être désapprouvée ou contestée par l'institution, bien au contraire. En effet, comme nous l'avons souligné en introduction, différents auteurs s'entendent pour dire que Cole lui-même serait à l'origine de cette appellation. Il la réemploie d'ailleurs publiquement lorsqu'il prononce son discours What is Art Culture? devant la Manchester School of Art en 1877 afin de commenter positivement l'effet saisissant de cette mise en exposition alors démantelée depuis près de vingt-cinq ans ${ }^{26}$.

Comme l'ont aussi remarqué Frayling et Kriegel, l'emploi de cette formulation accrocheuse fait très certainement référence à une autre " chambre des horreurs " célèbre : celle du musée de cire de Mme Tussaud alors situé sur Baker Street ${ }^{27}$. Dès 1802, année où elle quittait la France pour exhiber ses portraits de cire en Angleterre, Marie Tussaud présentait, dans une salle isolée de l'espace principal d'exposition, des portraits de criminels et autres condamnés à mort moulés sur des modèles encore vivants ou déjà exécutés (fig. 4). Dans cette Adjoining Room, dont le surnom de "Chamber of Horrors » circule dans la presse au moins à partir de la fin des années $1830^{28}$, le visiteur pouvait voir des moulages de têtes guillotinées pendant la Révolution française. Rapidement, de plus en plus de portraits de criminels anglais, parfois mis en scène dans des «tableaux » les présentant au moment de commettre leur crime ou lors de leur mise à mort, furent intégrés à cette exposition qui était complétée par des instruments de torture variés 29 .

La référence aux cires de Mme Tussaud n'est certainement pas un hasard. En avril 1850, deux ans avant l'ouverture de la Marlborough House, le décès de Mme Tussaud avait entraîné la parution de nombreux articles élogieux dans les journaux, et la " chambre des horreurs ", attraction la plus populaire de cette institution renommée, avait fait l'objet d'un agrandissement substantiel en prévision d'un achalandage accru lié à l'Exposition universelle de $1851^{30}$. L'intitulé de cette salle ainsi que son contenu devaient donc être connus par un large public, et son emploi en lien avec le Museum of Ornamental Art a toutes les apparences d'un coup publicitaire visant à susciter la curiosité des visiteurs.

Le lien établi entre le contenu des deux salles d'exposition par le biais du surnom " chambre des horreurs " est révélateur de la valeur accordée aux principes de bon design au Museum of Ornamental Art. Plus que de simples principes à suivre dans la conception et la réalisation d'un objet, ces principes sont élevés au rang de lois (laws) - terme d'ailleurs employé par Henry Cole dans son introduction au catalogue de 185331 - dont le nonrespect est passible de sanction de la part de l'institution. Ainsi, comme le suggère un journaliste selon qui, «Respectable families stared to see their pet article of ornament or item of gentility

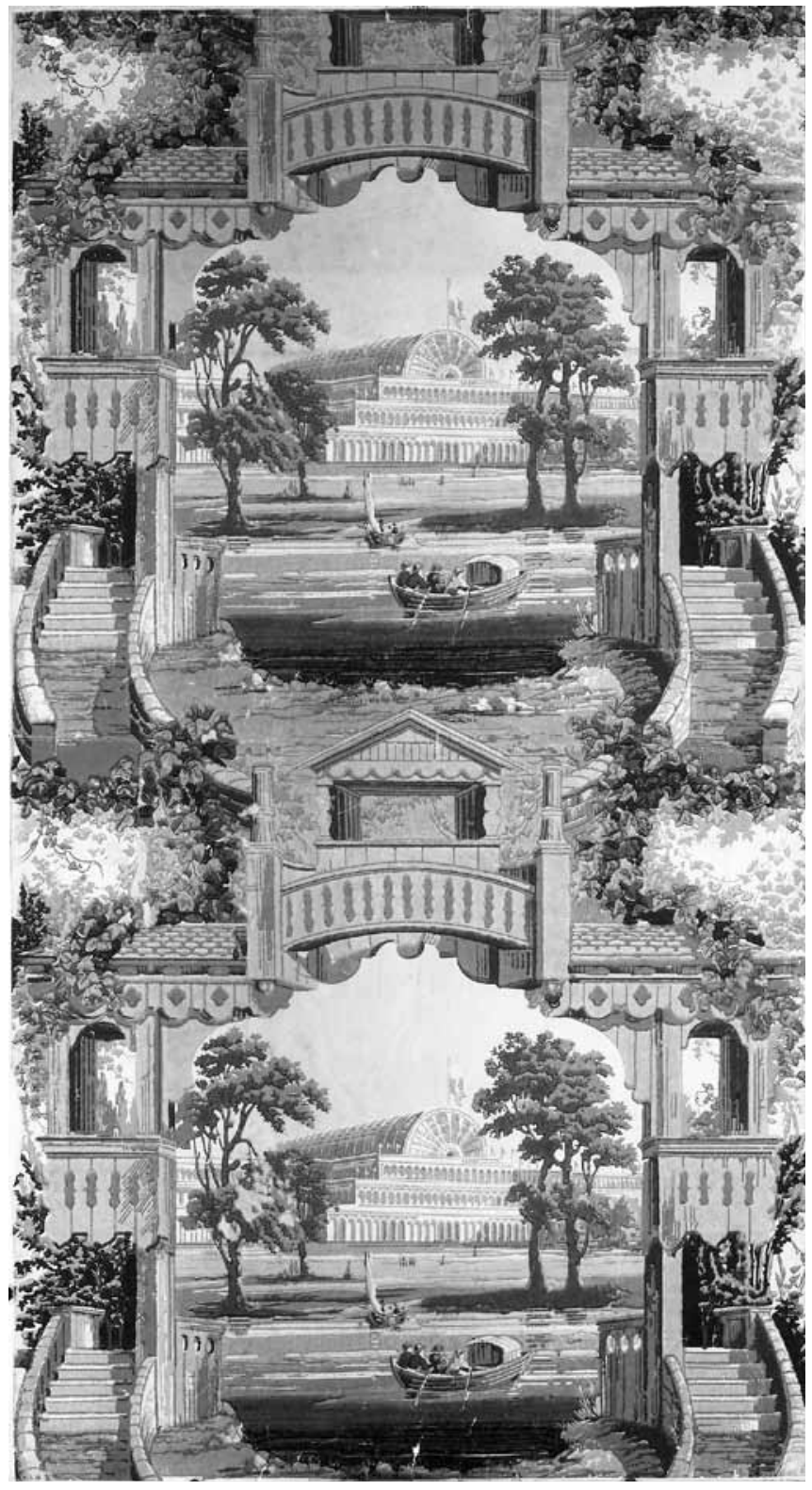

Figure 2. Heywood, Higginbottom \& Smith (?), « Perspective Representation of the Crystal Palace and Serpentine », vers 1853-55, papier peint, fragment $99 \times 53,6 \mathrm{~cm}$, Londres, Victoria and Albert Museum (@ Victoria and Albert Museum, London).

False Principle no 28 : Observations.-Perspective representation of the Crystal Palace and Serpentine; with flights of steps and architectural framework, causing the same error as in 27. A Catalogue of the Museum of Ornamental Art at Marlborough House, p. 17. 


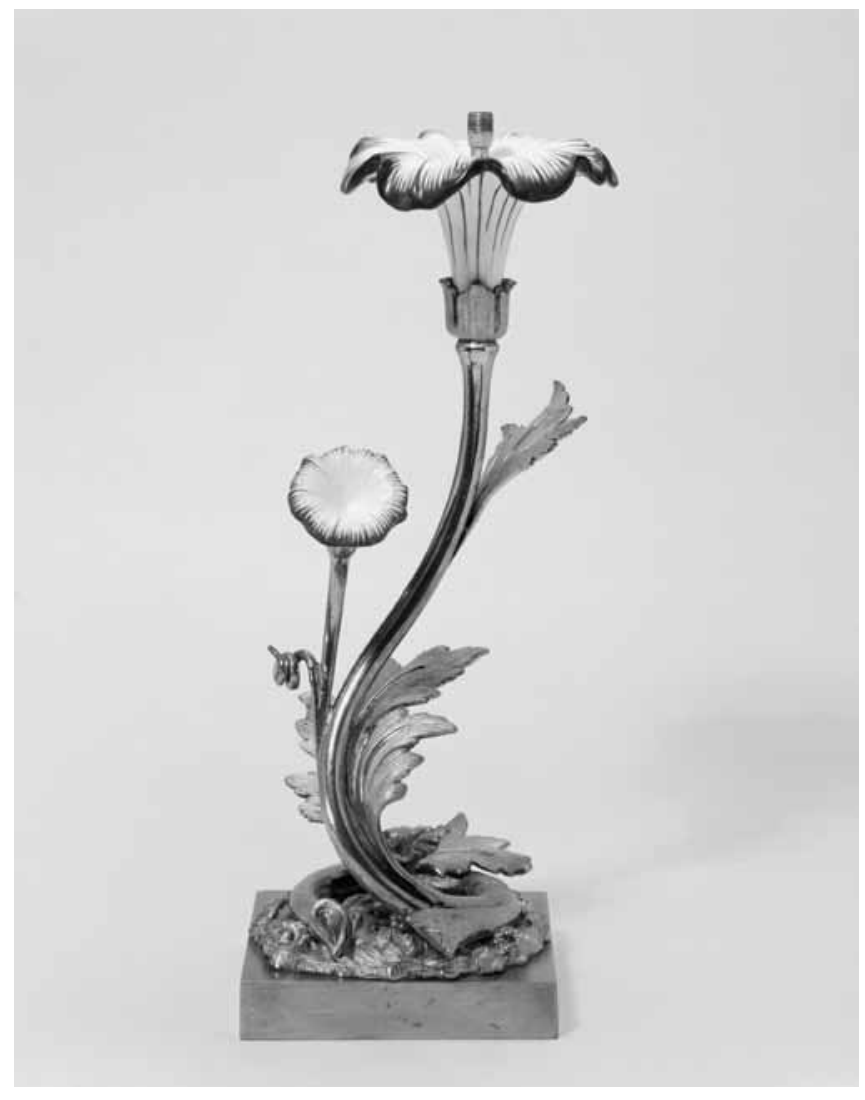

Figure 3. R. W. Winfield \& Co., 1848, lampe au gaz, cuivre doré et verre, $31.5 \times 14 \mathrm{~cm}$, Londres, Victoria and Albert Museum (@) Victoria and Albert Museum, London).

False Principle no 83 : Observations.-Gas flaming from the petal of a convolvulus! - one of a class of ornaments very popular, but entirely indefensible in principle. A Catalogue of the Museum of Ornamental Art at Marlborough House, p. 23.

gibbeted for public reprobation [...] »32, en les exposant dans la " chambre des horreurs ", le musée condamnerait à la potence des objets jugés « criminels » sur le plan esthétique. Par analogie, ces derniers acquièrent un statut semblable à celui des cadavres de cire de Mme Tussaud, ces auteurs de crimes condamnés à la peine capitale qui sont mis en scène de manière à pérenniser l'événementialité et le spectacle de la mise à mort publique. La référence à Mme Tussaud mène donc à l'institutionnalisation de la criminalisation de l'ornement conçu d'après des principes de design jugés mauvais par le musée et constitue la première formulation d'une attaque aujourd'hui indissociable des discours sur le design.

En 2002, dans Design and Crime, Hal Foster critiquait l'omniprésence du design contemporain dont l'inflation serait due à sa récupération par le système économique actuel. Pour l'auteur, le capitalisme récupère en les pervertissant les idéaux originellement émancipateurs - notamment la réconciliation entre l'art et la vie et le croisement des disciplines - des mouvements d'avant-garde artistique comme le Bauhaus, de manière à imposer l'homogénéisation et l'indistinction caractéristiques du design total33. Le titre et le contenu de cette diatribe reprenaient en les approfondissant les idées énoncées près d'un siècle plus tôt dans différents écrits d'Adolf Loos, dont Crime et Ornement (c. 1910) ${ }^{34}$. Dans ce texte, Loos condamnait l'omniprésence et l'abus de l'ornement, notamment dans les objets d'usage quotidien qui doivent demeurer distincts des œuvres d'art. Considéré comme une forme de gaspillage (du temps de travail et de l'énergie des ouvriers) et une profanation du matériau, l'ornement était pour Loos un signe de dégénérescence contraire à "l'évolution de la culture ", d'où sa dimension criminelle. Loos prônait l'adéquation entre la forme et la fonction de l'objet et le respect du matériau à travers le dépouillement absolu, comme en témoigne l'affection qu'il porte à son étui à cigarettes parfaitement " lisse »35.

Il n'existe pas de lien de cause à effet entre les positions de Cole, Loos et Foster, et leurs critiques respectives ne sont pas unies par une trajectoire téléologique. Soulignons toutefois que la fortune critique de Crime et Ornement, l'un des textes les plus connus et les plus cités de Loos, assure la diffusion de l'idée de la criminalisation de l'ornement tout au long du vingtième siècle. Précisons également que, parmi les nombreuses sources ayant inspiré Loos pour l'écriture de cette critique, Christopher Long note The Grammar of Ornament (1856) d'Owen Jones ${ }^{36}$. Les trente-sept principes de base énoncés au début de cet ouvrage sont presque tous identiques à ceux présentés par Jones lors de ses conférences intitulées On the True and the False in the Decorative Arts tenues en juin 1852 à la Marlborough House alors qu'il collaborait étroitement avec Henry Cole à l'élaboration du Museum of Ornamental Art, de ses collections, de son catalogue et, à n'en point douter, de sa " chambre des horreurs »37. Aussi, nous suggérons que la " chambre des horreurs » constitue une prise de position institutionnelle précoce qui doit être inscrite dans le contexte plus inclusif de l'histoire - ou peut-être la préhistoire - de la théorie du design dans laquelle la notion de criminalisation est présente. Chez Loos comme chez Foster, l'enjeu de cette criminalisation est celui de la distinction. Tous deux attaquent la notion d'œuvre d'art totale (gesamtkunstwerk) dont la complétude tend à effacer les limites et instaure une forme d'indistinction qui mène à la fusion de l'objet (l'œuvre ou le produit) et du sujet (l'individu). Pour les deux auteurs, le principe de l'œuvre d'art totale conduit à une apothéose du sujet qui, paradoxalement, est synonyme de sa mort dans la mesure où c'est justement la distinction qui permet de ménager la " marge » nécessaire à l'individu pour désirer, rêver, exister ${ }^{38}$.

Avec la " chambre des horreurs", le Museum of Ornamental Art propose une critique moins radicale de l'ornement et 


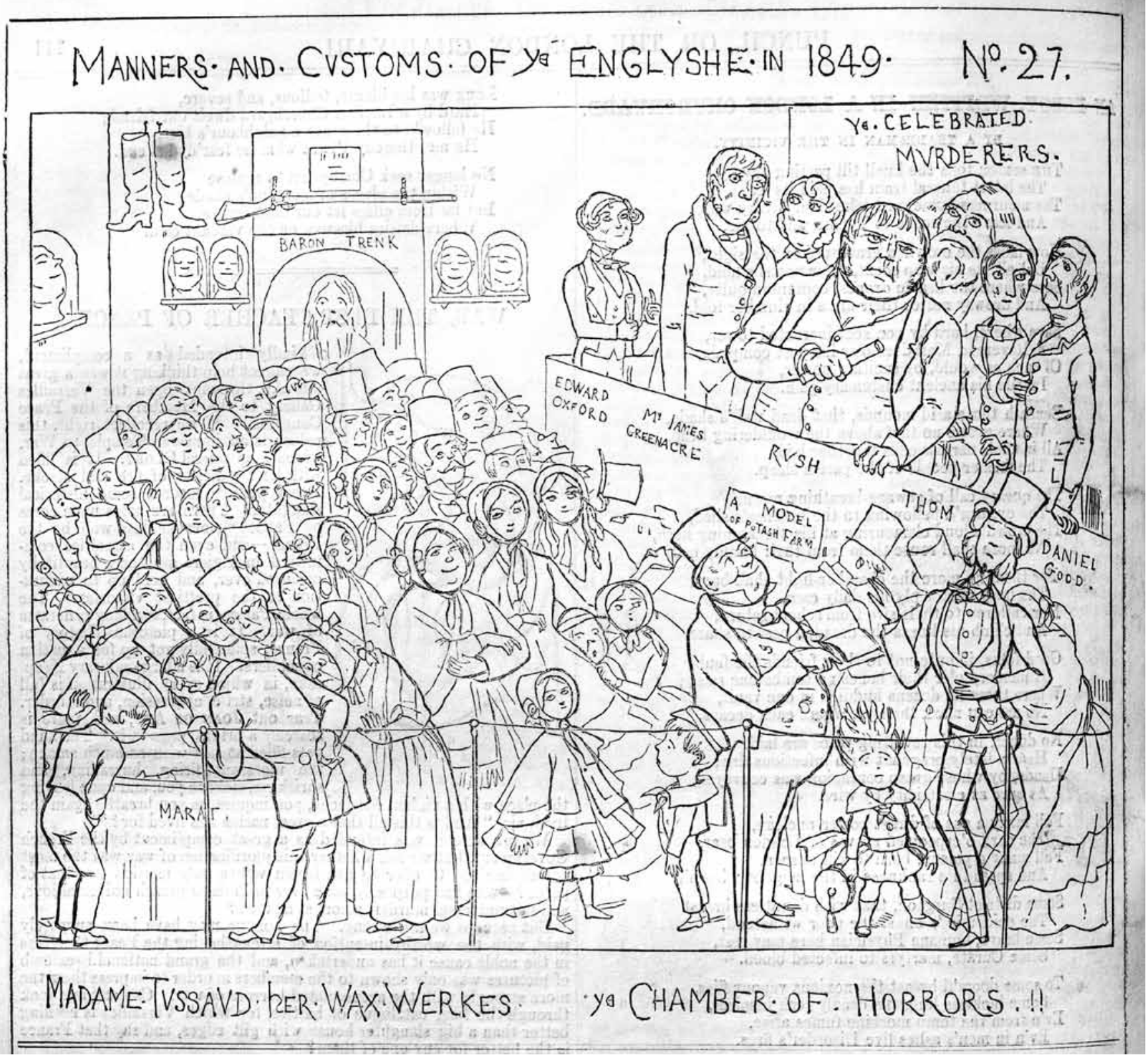

Figure 4. « Manners and Customs of Ye Englyshe in 1849, № 27-Madame Tussaud her Wax Werkes Ye Chamber of Horrors!! », Punch, The London Charivari, vol. 16, 1849 (Livres rares et collections spécialisées, Bibliothèque de l'université McGill).

cible surtout son usage extravagant en regard des principes de bon design qu'il tente d'instituer. Mais, puisque ces principes participent à la construction de la spécificité de l'art ornemental depuis une perspective fonctionnaliste, il s'agit ici aussi d'une critique visant à introduire une distinction - et donc une marge - par rapport aux « beaux arts ». À ce titre, rappelons qu’au milieu du dix-neuvième siècle, la signification de « design » en tant que mode de conception de l'objet adapté aux réalités de la production de masse est justement en cours d'élaboration. L'institutionnalisation par le musée d'objets produits en multiples et considérés comme appartenant aux arts mécaniques, appliqués, décoratifs ou industriels selon le contexte et l'époque ${ }^{39}$, contribue à créer la notion de design en officialisant à la fois le statut artistique de ces objets et leur séparation des beaux arts, et en constituant par là même le canon par rapport auquel leur valeur esthétique sera jugée ${ }^{40}$. Toutefois, comme nous le 
démontrerons, si les moyens initialement employés au Museum of Ornamental Art pour faire valoir cette distinction sont inefficaces, c'est notamment en raison de leur ambiguïté qui, ironiquement, relève d'une forme d'indistinction.

Les principes de bon design et leurs contraires : l'exemplarité problématique de l'objet dans la « chambre des horreurs »

Les objets exposés dans l'ensemble du Museum of Ornamental Art étant jugés comme des exemples de biens conçus et réalisés selon les principes de bon design, ceux présentés dans la " chambre des horreurs" peuvent être considérés comme des contre-exemples. Si cette expression n'est pas textuellement employée par l'instance muséale, le fait que Cole décrive ces objets comme étant remarquables uniquement parce qu'ils dérogent à tous les principes et même au sens commun le plus évident en ce qui concerne leur décoration nous permet de les identifier comme tels ${ }^{41}$. Toutefois, le recours à l'exemple et à son contraire comme stratégie pédagogique au musée est problématique, car son efficacité repose sur une opposition binaire nécessitant une forte polarisation. L'intelligibilité de ce type d'exposé requiert la simplification, voire la réduction des concepts qui y sont enseignés, surtout dans le cas d'un discours dont l'argumentaire se déploie visuellement, incarné dans les objets.

En plus de montrer que les responsables du Museum of Ornamental Art sont convaincus de la légitimité des principes de bon design, le recours à la « chambre des horreurs " témoigne de leur confiance dans le pouvoir auto-explicatif de la présentation d'un argument sur le mode visuel à travers sa mise en exposition. Cependant, l'efficacité visuelle du message véhiculé par la présentation de contre-exemples ne va pas de soi. En effet, un auteur anonyme rapporte dans The Observer l'incompréhension d'un groupe de jeunes filles devant un mouchoir de coton, probablement l'objet numéro 37 de la "chambre des horreurs "42. Selon les explications données dans le Catalogue of the Museum of Ornamental Art, l'objet est condamné en raison de son motif décoratif décrit comme étant grossier, aux couleurs inharmonieuses et ne convenant pas à son usage ${ }^{43}$. Toutefois, c'est plutôt la signification de la devise "Honesty is the Best Policy " inscrite sur le mouchoir que les jeunes filles semblent associer à sa condamnation. Cette anecdote démontre que présenter un argument visuellement ne le rend pas nécessairement intelligible. Sans les explications complémentaires du catalogue, l'argument esthétique qui préside à la classification du mouchoir dans la " chambre des horreurs " est perverti en un jugement moral.

Henry Cole est conscient du fait que le mode visuel ne suffit peut-être pas à assurer la transmission des principes de bon design. S'il soutient que l'organisation adéquate du musée peut le rendre instructif au plus haut point, il admet néanmoins que le recours à d'autres stratégies en accroîtra l'intelligibilité. Selon lui :

By proper arrangements a Museum may be made in the highest degree instructional. If it be connected with lectures, and means are taken to point out its uses and applications, it becomes elevated from being a mere unintelligible lounge for idlers into an impressive schoolroom for everyone ${ }^{44}$.

La production du catalogue serait ainsi l'un des moyens employés par Cole pour transformer le musée en une salle de classe accessible à tous. En plus d'y retrouver les principes de design fondamentaux, le visiteur y lit une courte description de chaque objet rédigée uniquement en fonction de ces règles. Toutefois, si la combinaison de l'illustration par l'objet (le visuel) et des explications (le textuel) semble être la solution pour garantir la qualité de l'enseignement, elle en révèle aussi la dimension tautologique. Comme le souligne l'auteur de l'article déjà cité publié dans The Times le 6 septembre 1852, en donnant les raisons qui justifient la condamnation des objets montrés dans la " chambre des horreurs », le musée offre au public l'opportunité de vérifier la justesse des canons qu'il enseigne ${ }^{45}$. Ainsi, l'objet présenté au musée doit être examiné à partir des principes de design énoncés dans le catalogue, et l'examen de l'objet permettra en retour de valider l'exactitude de ces mêmes principes. L'autorité du musée est construite à travers un argument circulaire et le catalogue devient davantage un outil de légitimation du dogmatisme esthétique de l'institution qu'un support didactique.

Le catalogue, vendu au coût de trois ou six pence selon les éditions, semble avoir suscité un certain intérêt : près de 11,5\% des personnes ayant visité le musée au cours des vacances de Noël de l'année $1852^{46}$ se procurent un exemplaire et une cinquième édition est produite dès mai 1853. Malgré cette relative popularité, il y a lieu de se demander quelle proportion des visiteurs lit avec attention l'ensemble des onze pages consacrées aux objets présentés dans la chambre des horreurs. De plus, il est impossible de connaittre l'usage réel qu'en font les visiteurs lors de leur visite au musée et encore moins leur niveau de compréhension des enjeux présentés par l'exposition.

Une gravure qui accompagne le court article intitulé "Sevres and other porcelains at the Exhibition of Art Manufactures " publié dans The Illustrated London News en 1852 donne cependant un indice (fig. 5); elle semble suggérer la faible incidence voire l'insuccès du catalogue comme outil d'interprétation des œuvres. Cette gravure serait la plus ancienne représentation de la mise en exposition des objets à la Marlborough House et, à notre connaissance, elle n'a pas encore fait l'objet d'un commentaire détaillé. L'enfilade des pièces perceptible sur la droite de l'image laisse supposer qu'il s'agit de la salle 20 où sont présentées les poteries conformément au plan 


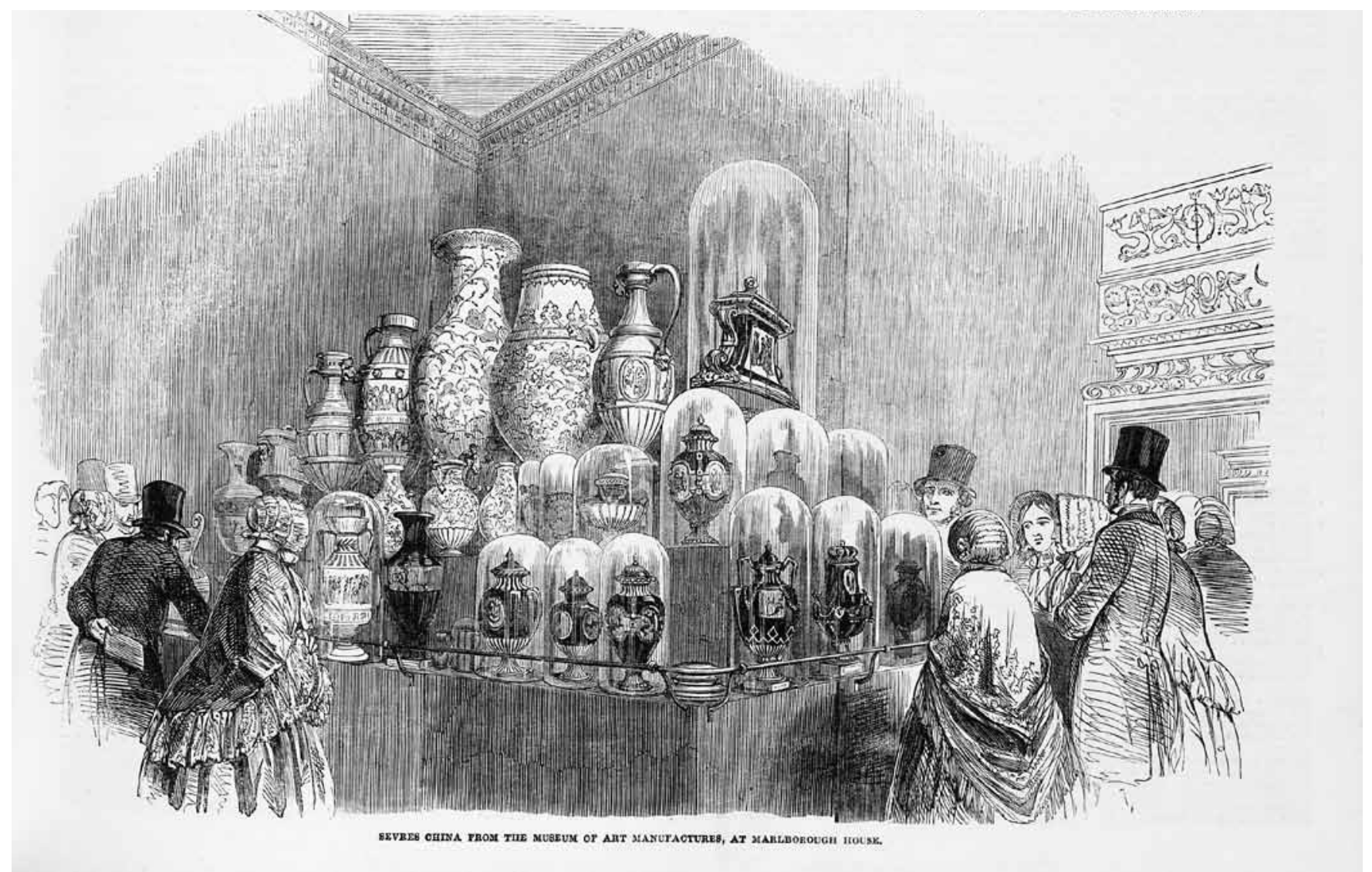

Figure 5. «Sevres China from the Museum of Art Manufactures, at Marlborough House », The Illustrated London News, vol. 2I, n 579 , semaine se terminant le 18 septembre 1852, p. 22 I (Livres rares et collections spécialisées, Bibliothèque de l'université McGill).

(fig. 1). Cette interprétation est corroborée par la description du contenu des salles d'exposition du musée consignée dans le First Report of the Department of Practical Art ${ }^{47}$. En raison de leur taille et de leur somptuosité, les porcelaines représentées dans la gravure sont très probablement celles de la reine Victoria qui prête au musée une quarantaine de pièces de porcelaine de Sèvres en provenance de sa collection personnelle conservée au Palais de Buckingham ${ }^{48}$. Les visiteurs, femmes coiffées d'un bonnet et aux épaules recouvertes d'un châle et hommes portant le haut-de-forme, se pressent les uns contre les autres pour observer les objets regroupés sur un présentoir à gradins. Sur le plan visuel, la présentation pyramidale domine les visiteurs et tend à magnifier les céramiques tandis que la présence de cloches de verre souligne leur préciosité et contribue à élever leur statut au rang d'" objet idéal ». Sur le plan textuel, un cartel allongé est visible sur la base du présentoir du côté droit, mais il est impossible de savoir s'il s'agit d'une énumération des œuvres présentées ou d'une explication des principes de bon design. Nous remarquons par contre qu'un seul des visiteurs, un homme situé à la gauche de la composition, tient dans sa main ce qui a l'apparence du catalogue d'exposition.

Comme le suggère cette gravure, l'attrait visuel des objets semble susciter davantage d'intérêt que les explications écrites. Pourtant, tel que le souligne le First Report of the Department of Practical Art, bien que les porcelaines de Sa Majesté illustrent l'excellence des qualités techniques atteintes par la Manufacture de Sèvres au cours des dix-septième et dix-huitième siècles, elles ne doivent pas être considérées comme des exemples à suivre au point de vue de la forme ${ }^{49}$. Ce reproche substantiel n'est pas explicité davantage, ce qui constitue à notre avis un symptôme de l'arbitraire de la distinction entre le bon et le mauvais design ainsi que de la confusion qu'elle engendre dans le contexte de l'exposition du Museum of Ornamental Art.

Qui plus est, le fait que la disparité entre l'excellence technique et la piètre qualité formelle des porcelaines ne soit pas prise en compte dans les articles publiés dans la presse tend aussi à confirmer le peu d'attention portée aux informations contenues dans le catalogue. En effet, les comptes rendus de 
l'exposition insistent uniquement sur la somptuosité de ces pièces et sur leur coût élevé, mais ne mentionnent pas que leur conception déroge aux principes de bon design ${ }^{50}$. C'est notamment le cas de l'article qui accompagne la gravure illustrée ici. L'ensemble des porcelaines y est décrit comme l'un des éléments les plus intéressants de l'exposition et les prix élevés de certaines pièces sont justifiés par le fait qu'elles unissent les triomphes de la chimie, les habiletés exquises des modeleurs et l'excellence des peintres ${ }^{51}$. Non seulement l'article ne mentionne pas que ces objets exposés hors de la " chambre des horreurs " ne sont pas conçus en parfait accord avec les principes de bon design, la gravure véhicule le message contraire. Elle représente une muséographie qui n'encourage pas un examen critique où la valeur technique serait considérée indépendamment de la forme et du décor.

Notre analyse fait valoir qu'une muséographie basée sur l'opposition visuelle entre exemple et contre-exemple rend difficilement compte des nuances. Serait-ce dû - comme nous le verrons dans la section qui suit - au fait que le musée en lui-même, de par son autorité, prédispose à l'admiration, peu importe ce qu'il montre ? Toujours est-il que l'objet qui n'est pas entièrement " bon " ou entièrement " mauvais " se prête difficilement à un tel exercice, ce qui montre que l'instrumentalisation de l'œuvre au profit de l'édification du discours muséal comporte des limites. Bien marquée en théorie, l'opposition binaire entre bons et mauvais principes de design est beaucoup plus subtile en pratique, et il est parfois difficile de distinguer les bons des mauvais objets au point où certains semblent interchangeables dans le musée. Frayling remarque lui aussi que certains des objets présentés au Museum of Ornamental Art sont conçus selon des principes de design qui pourraient être qualifiés à la fois de bons et de mauvais. Il mentionne que l'inadéquation entre l'excellence technique de la réalisation et la qualité de la conception suscite des discussions entre les membres du comité d'acquisition dès $1851^{52}$. Le cas ambigu des porcelaines royales n'est donc pas isolé.

Par exemple 53 , l'objet numéro 61 de la « chambre des horreurs ", une assiette à dessert en porcelaine réalisée à Sèvres, est condamnée parce que les fleurs peintes au centre ne seront plus visibles au moment d'en faire usage (l'ornementation n'est donc pas en harmonie avec la fonction de l'objet) et parce que le décor coloré masque la blancheur spécifique du matériau ${ }^{54}$. Dans la section du catalogue dédiée à la salle "Decorations on False Principles ", Richard Redgrave souligne que le centre des assiettes et autres vaisselles doit être libre de tout décor pour des raisons d'utilité, mais aussi de propreté55. Les explications semblent claires et le contre-exemple parait efficace. Mais, une fois sorti de la "chambre des horreurs ", le visiteur sera confronté à une autre assiette à dessert, soit la pièce numéro 54 . D'après la description du catalogue, cette pièce produite par Minton \&
Co. est décorée de fleurs et d'un cupidon peint au centre ${ }^{56}$. Bien que présentée à titre d'exemple des principes de bon design, cette assiette, dont le décor n'est pas critiqué, enfreint la même règle que l'assiette numéro 61 qui est pourtant exposée dans la " chambre des horreurs".

Parmi les nombreuses pièces produites à Sèvres et exposées dans le musée se trouvent également les vases numéro 25 et 26 de la division "Pottery " du catalogue du Museum of Ornamental Art de 1852. Ceux-ci auraient été choisis en raison de l'excellence de leur exécution mais pas pour la qualité de leur design ${ }^{57}$. Pourquoi le statut de ces objets est-il différent de celui de l'assiette numéro 61 ? Produite à Sèvres, n'était-elle pas, elle aussi, un exemple de grande maîtrise technique ? L'inadéquation entre la qualité de la réalisation et celle du design complique d'autant plus l'éducation esthétique du visiteur qu'elle n'est pas à sens unique. Certains objets sont en effet considérés comme étant des exemples de bon design bien que la piètre qualité de leur réalisation ne soit pas un exemple à imiter. C'est le cas de la coupe numéro 21 présentée dans la section poterie et dont les ornements sont jugés élégants malgré une exécution rude ${ }^{58}$. L'interchangeabilité potentielle de ces objets dans le dispositif muséographique met non seulement en péril la transmission visuelle du message, elle sème le doute quant à l'autorité du discours véhiculé par l'exposition.

\section{De la confusion à la contamination, ou l'échec de la « chambre des horreurs »}

La présence de la "chambre des horreurs » au musée implique un double phénomène de contamination qui, en définitive, scelle son impossibilité. D'une part, l'autorité de l'institution muséale rend inaudible sa dénonciation de certains objets. Comme le souligne Chantal Georgel, derrière le dévouement didactique du musée du dix-neuvième siècle se trouve " une préoccupation profonde, rarement inconsciente : celle de "moraliser" la société "59. Le musée institutionnalise ainsi le topos de l'adéquation entre la morale et la beauté. En tant que " temple du beau et du bien ", il prédispose le visiteur à une expérience esthétique peu critique où le contraire du beau et du bien - dans ce cas-ci le contenu de la « chambre des horreurs " - a difficilement sa place. Le musée contamine donc lui-même la " chambre des horreurs " alors que les objets qui y sont présentés risquent d'être considérés à tort comme des exemples de bon design.

D'autre part, parce que sa valeur de contre-exemple est peu probante, la « chambre des horreurs » mine de l'intérieur la légitimité du discours institutionnel et subvertit potentiellement l'ensemble des collections, inversant ici le processus de contamination. Cette idée est poussée au seuil du ridicule dans la presse alors que le risque de contamination est justement l'une des critiques formulées par les détracteurs de la chambre des horreurs 
du musée de Mme Tussaud. Dans un article du journal satirique Punch, un père de famille fictif clame son désespoir depuis que sa fille s'est prise d'affection pour des meurtriers après avoir vu leur effigie de cire chez Mme Tussaud ${ }^{60}$. Un autre auteur qualifie ces mêmes criminels de "poison moral " et soutient que la chambre des horreurs risque de contaminer non seulement Baker Street mais Londres en entier ${ }^{61}$. Plus insidieusement, la " chambre des horreurs » du Museum of Ornamental Art, elle, mettrait en péril la santé mentale des visiteurs. La contamination morale devient pathologie. C'est le triste cas de M. Crumpet, héros de la nouvelle satirique écrite par Henry Morley, "A House Full of Horrors ». Traumatisé suite à une visite à la Marlborough House, le personnage prend conscience qu'il vit entouré d'horreurs. Obsédé par ses nouvelles connaissances au sujet des principes de bon design, Crumpet atteint un état "d'apoplexie mentale »62. L'horreur des objets du quotidien les plus simples lui est désormais insupportable, au point où il en perd presque la raison. Bien que caricaturaux, ces cas révèlent le potentiel de perversion attribué à la seule présentation du contre-exemple dans la " chambre des horreurs".

Par ailleurs, la situation de la " chambre des horreurs » dans le parcours muséal et sa grande popularité contribuent à en faire un agent de subversion et ce, malgré le rôle pédagogique qui lui est attribué par le Museum of Ornamental Art. Non seulement cette salle amorce-t-elle la visite de l'exposition, moment où la concentration des visiteurs est généralement la plus soutenue, il est impossible de l'éviter - contrairement à la " chambre des horreurs " de Mme Tussaud située en retrait de l'exposition principale et accessible après avoir déboursé un supplément de six pence ${ }^{63}$. Vus en premier, les mauvais principes de design devraient servir de repoussoir et ainsi mettre en valeur le reste des objets de la collection. Mais, comme en témoigne le succès de la " chambre des horreurs ", il est tout à fait plausible que les contre-exemples, aussi ambigus et discutables soient-ils, deviennent plutôt des points de repère formant le cadre de référence à partir duquel se fera l'examen de chaque objet présenté au musée. En effet, un extrait du First Report of the Department of Practical Art souligne que cette salle suscite un intérêt beaucoup plus considérable que plusieurs objets dont l'excellence n'est généralement pas appréciée par les visiteurs ${ }^{64}$. Nous constatons ici un double échec : non seulement le contre-exemple est peu efficace, il retient l'attention du visiteur au détriment des objets jugés acceptables, voire se substitue à eux.

\section{La mort annoncée de la « chambre des horreurs »}

En prônant la criminalisation de l'ornement comme principe moteur de la salle "Decorations on False Principles ", le musée annonçait ainsi, quoique sans vouloir l'admettre, la mort de cette mise en exposition. Cette impasse incarne en quelque sorte la tautologie de l'argument muséal par rapport aux principes de bon design. L'analyse de la chambre des horreurs permet ainsi d'éclairer certaines modalités de la relation de pouvoir que le musée en tant qu'institution établit avec le public et de cerner les limites des moyens muséographiques dont il dispose pour transmettre son message et faire valoir son autorité.

Par ailleurs, l'ambition du musée d'instaurer et d'inculquer des principes de bon design de manière à marquer la spécificité de l'art ornemental trouvera des échos dans les plaidoyers de Loos et de Foster en faveur de la distinction. En tant qu'expression ultime de cette ambition, la " chambre des horreurs " s'impose donc comme un précédent archéologique inatten$\mathrm{du}$, un cas unique mais révélateur qui permet d'approfondir l'examen critique des paradoxes associés à la notion de criminalisation dans l'élaboration des théories du design. Aussi, la stratégie de la " chambre des horreurs " nous rappelle que, même chez ceux qui continuent à le défendre, l'ornement peut être criminel.

\section{Remerciements}

Je tiens à remercier Johanne Lamoureux pour ses précieux conseils ainsi que Ginette Jubinville pour ses lectures des différentes versions de ce manuscrit. Merci également à Ersy Contogouris, aux rédacteurs de $R A C A R$ et aux réviseurs externes pour leurs commentaires et suggestions.

Notes

1 En mai et juin 1852, le musée ouvre temporairement ses portes pendant dix-sept jours suite auxquels les collections seront réorganisées en vue de l'ouverture officielle. Christopher Frayling, Henry Cole and the Chamber of Horrors. The Curious Origins of the Victoria and Albert Museum, Londres, 2010, p. 27. Notons en outre qu'au cours de l'année 1852, le Museum of Ornamental Art est d'abord nommé Museum of Manufactures, intitulé que nous avons choisi de ne pas employer dans le texte afin d'éviter toute confusion.

2 Cette thèse est soutenue par Frayling, Henry Cole and the Chamber of Horrors, p. 28, et par Elizabeth Bonython et Anthony Burton, The Great Exhibitor. The Life and Work of Henry Cole, Londres, 2003, p. 154.

3 "Museum of Ornamental Art ", The Times, 6 septembre 1852, p. 4.

4 "Examples of False Principles in Decoration ", dans A Catalogue of the Museum of Ornamental Art at Marlborough House, Londres, 1853, p. 13.

5 Deborah Cohen, Household Gods. The British and Their Possessions, New Haven et Londres, 2006, p. 24 ; Frayling, Henry Cole and the Chamber of Horrors, p. 67-8. Pour sa part, Lara Kriegel suggère que sa fermeture pourrait être liée à des contraintes d'espace dans le musée. Lara Kriegel, Grand Designs. Labor, Empire and the Museum 
in Victorian Culture, Durham et Londres, 2007, p. 148. Voir aussi "The Museum of Ornamental Art ", The Spectator, 22 octobre 1853, p. 1022.

6 Avec l'idée d'ornement criminel nous faisons référence au texte d'Aldolf Loos Crime et Ornement (c. 1910) auquel nous reviendrons ultérieurement.

7 Voir notamment John Physick, The Victoria and Albert Museum : The History of Its Building, Oxford, 1982 ; Malcom Baker et Brenda Richardson (dir.), A Grand Design: The Art of the Victoria and Albert Museum, New York et Baltimore, 1997 ; Anthony Burton, Vision \& Accident. The Story of the Victoria and Albert Museum, Londres, 1999.

8 Suga Yasuko, "Designing the Morality of Consumption : "Chamber of Horrors" at the Museum of Ornamental Art, 1852-53 ", Design Issues, vol. 20, $\mathrm{n}^{\circ}$ 4, 2004, p. 43-56.

9 Fondée en 1837, la School of Design occupe d'abord Somerset House avant d'être déménagée à Marlborough House en 1852.

10 Pour plus de détails au sujet de l'histoire des collections du Victoria and Albert Museum, voir Anna Somer Cocks, The Victoria and Albert Museum : The Making of the Collection, Leicester, 1980 ; Clive Wainwright, "The Making of the South Kensington Museum I ", "The Making of the South Kensington Museum II ", "The Making of the South Kensington Museum III ", et "The Making of the South Kensington Museum IV ", Journal of the History of Collections, vol. 14, $\mathrm{n}^{\circ} 1,2002$, p. 3-23, 25-44, 45-61, et 63-78.

11 Baker et Brenda (dir.), A Grand Design; Burton, Vision \& Accident; Bonython et Burton, The Great Exhibitor.

12 Henry Cole, dans First Report of the Department of Practical Art, Londres, 1853, p. 33. Le Department of Practical Art deviendra le Department of Science and Art en 1853.

13 Cole, dans First Report of the Department of Practical Art, p. 2.

14 Clive Wainwright, "Principles True and False : Pugin and the Foundation of the Museum of Manufactures ", The Burlington Magazine, vol. 136, $\mathrm{n}^{\circ} 1095,1994$, p. 362.

15 Anthony Burton, "The Uses of the South Kensington Art Collections ", Journal of the History of Collections, vol. 14, $n^{\circ}$ 1, 2002, p. 81 ; Rafael Cardoso Denis, "Teaching by Example : Education and the Formation of South Kensington's Museums », dans Baker et Richardson (dir.), A Grand Design.

16 A Catalogue of the Museum of Ornamental Art, 5-8.

17 Voir par exemple, Charles Dickens, "Hard Times ", Household Words. A Weekly Journal conducted by Charles Dickens, vol. IX, $1^{\text {er }}$ avril 1854, p. 143.

18 A. W. N. Pugin, The True Principles of Pointed or Christian Architecture, Londres, 1841, p. 1.

19 Owen Jones, On the True and False in Decorative Arts. Lectures Delivered at Marlborough House, June 1852, Londres, 1863, p. 2.

20 A Catalogue of the Museum of Ornamental Art, p. 7.

21 Jones, On the True and False in Decorative Arts, p. 3. Cette idée est reprise par Cole, A Catalogue of the Museum of Ornamental Art, p. 5.

22 Museum of Ornamental Art. A Hand-Book Guide for Visitors, Londres, 1852, p. 3-32; A Catalogue of the Museum of Ornamental Art, p. 5-8

23 Cole, dans First Report of the Department of Practical Art, p. 33.

24 Kriegel, Grand Designs, p. 130.

25 A Catalogue of the Museum of Ornamental Art, p. 22-3.

26 Henry Cole, What is Art Culture? An Address Delivered to the Manchester School of Art, Manchester, 1877, p. 6.

27 Frayling, Henry Cole and the Chamber of Horrors, p. 28 ; Kriegel, Grand Designs, p. 145.

28 Pamela Pilbeam attribue la paternité de ce surnom au magazine Punch en 1846. Mais l'intitulé se retrouve déjà en 1837 dans un article de The New Monthly Magazine and Humorist. Nous ignorons si l'expression est employée plus tôt et qui l'associe au musée de cire de Mme Tussaud pour la première fois. Pamela Pilbeam, $M a-$ dame Tussaud and the History of Waxworks, Londres et New York, 2003, p. 108 ; Henry Brownriss, "Midnight at "Madame T.'s" ", The New Monthly Magazine and Humorist, vol. 49, 1837, p. 393.

29 Pilbeam, Madame Tussaud and the History of Waxworks, p. 66-68, 79, 108.

30 Pilbeam, Madame Tussaud and the History of Waxworks, p. 124-25, 157.

31 A Catalogue of the Museum of Ornamental Art, p. 5-7.

32 "The Museum of Ornamental Art ", The Spectator, p. 1022.

33 Hal Foster, "Design and Crime ", dans Design and Crime (And Other Diatribes), Londres, 2002, p. 13-26.

34 Au sujet de la datation du texte de Loos voir Christopher Long, "The Origins and Context of Adolf Loos's "Ornament and Crime" ", Journal of the Society of Architectural Historians, vol. 68, n 2, 2009, p. 200-23.

35 Adolf Loos, "Ornement et Crime (1908) ", dans Ornement et Crime, trad. Sabine Cornille et Philippe Ivernel, Paris, 2003, p. 71-87.

36 Christopher Long, "The Origins and Context of Adolf Loos's "Ornament and Crime" ", p. 207-09.

37 Voir Owen Jones, The Grammar of Ornament, Londres, 1856, p. 5-8 ; Jones, On the True and False in Decorative Arts, p. 4-69.

38 Foster, "Design and Crime ", p. 15-17, 25. Pour désigner la marge, Foster emprunte à Karl Krauss l'expression spielraum qu'il traduit par running-room.

39 À propos de l'interchangeabilité de ces expressions et de l'ambiguïté des notions " arts décoratifs " et " design ", voir Isabelle Frank, The Theory of Decorative Art. An Anthology of European and American Writings, 1750-1940, New Haven et Londres, 2000, p. 1-26.

40 Au sujet du rôle des musées pour la division entre les beaux arts et les arts décoratifs, voir Christopher Whitehead, Interpreting Art in Museums and Galleries, Londres et New York, 2012, p. 102.

41 A Catalogue of the Museum of Ornamental Art, p. 7. 
42 "The People at Marlborough House ", The Observer, 9 janvier 1853 , p. 5 . Yasuko relève aussi cet exemple, « Designing the Morality of Consumption ", p. 52.

43 A Catalogue of the Museum of Ornamental Art, p. 18.

44 Cole, dans First Report of the Department of Practical Art, p. 30.

45 "Museum of Ornamental Art", The Times, p. 4.

46 "The People at Marlborough House", The Observer, p. 5. Entre le 27 décembre 1852 et le 6 janvier 1853, 16359 personnes visitent le musée et 1827 exemplaires du catalogue sont vendus, ce qui permet d'évaluer à 11,5 le pourcentage des visiteurs ayant acheté un catalogue.

47 First Report of the Department of Practical Art, p. 386-87.

48 Le nombre est de 42 ou 44 et la durée de ce prêt n'est pas précisée. First Report of the Department of Practical Art, p. 32, 387.

49 First Report of the Department of Practical Art, p. 387.

50 Par exemple : "Museum of Ornamental Art ", The Times, p. 4 ; "The Museum of Ornamental Manufactures ", The Illustrated London News, vol. 21, $\mathrm{n}^{\circ} 578$, semaine se terminant le 11 septembre 1852, p. 195.

51 "Sevres and other porcelains at the Exhibition of Art Manufactures ", The Illustrated London News, vol. 21, no 579, semaine se terminant le 18 septembre 1852, p. 221.

52 Frayling, Henry Cole and the Chamber of Horrors, p. 28-9.

53 Il est extrêmement difficile de retracer les premiers objets des collections du Victoria and Albert Museum et certains ont même disparu, d'où l'absence malheureuse d'illustration pour appuyer cette portion de l'analyse.

54 A Catalogue of the Museum of Ornamental Art, p. 20. Bien qu'à ce jour cet exemple particulier n'ait pas été répertorié dans les collections du musée, il est aisé d'imaginer ce genre d'assiette au marli orné et au centre fleuri - ou encore décoré d'une vue, ce qui serait tout aussi condamnable - en provenance des nombreux services de Sèvres.

55 A Catalogue of the Museum of Ornamental Art, p. 19-20.

56 Museum of Ornamental Art. A Hand-Book Guide for Visitors, p. 25.

57 Museum of Ornamental Art. A Hand-Book Guide for Visitors, p. 24.

58 Museum of Ornamental Art. A Hand-Book Guide for Visitors, p. 23.

59 Chantal Georgel, «Le musée, lieu d'enseignement, d'instruction et d'édification ", dans La jeunesse des musées. Les musées de France au XIXe siècle, catalogue d'exposition, Paris, Musée d'Orsay, Chantal Georgel (dir.), Paris, 1994, p. 66.

60 "Horrid Murder in Baker Street ", Punch, or the London Charivari, vol. 17, 1849, p. 123.

61 "Horrid Murder in Baker Street », p. 123.

62 [Henry Morley], "A House full of Horrors ", Household Words. A Weekly Journal conducted by Charles Dickens, vol. VI, 4 décembre 1852, p. 270.

63 Pilbeam, Madame Tussaud and the History of Waxworks, p. 79 et 112.

64 Cole, dans First Report of the Department of Practical Art, p. 33. 